\title{
A Look in the Mirror: How the Field of Behavior Analysis Can Become Anti-Racist
}

Sonia Levy ${ }^{1}$, Amy Siebold $^{2}$, Janani Vaidya ${ }^{3}$, Marie-Michèle Truchon ${ }^{2}$, Jamine Dettmering ${ }^{2}$, and Cameron Mittelman²

${ }^{1}$ Impact Village, Ajax, Ontario, Canada

${ }^{2}$ Department of Applied Behavior Analysis, The Chicago School of Professional Psychology

${ }^{3}$ Louisiana Contextual Science Research Group, Lafayette, Louisiana

\section{Authors Note}

Please address any correspondence to Sonia Levy at sonia.levy44@gmail.com or 289980-8446.

\section{Abstract}


BEHAVIOR ANALYSIS AND ANTI-RACISM

Sparked by recent events, discussions of systemic racism and racial inequalities have been pushed to the foreground of our global society, leading to what is being called the largest modern-day civil rights movement (Buchanan et al., 2020). In the past, Black, Indigenous, and People of Color (BIPOC) activists and scholars, amongst others, have evaluated and critiqued systems and organizations within our society. Nonetheless, it was not until recently that this movement was truly noticed by a greater number of people, some of whom are now further assessing how BIPOC are viewed and treated within their organization and by society as a whole (Worland, 2020). This is not only due to the increase in video evidence (e.g., released body cam footage, social media postings), but also to the previous administration's rhetoric and political agenda (Hubler \& Bosman, 2021). Police departments, educational institutions, and large companies have, for decades, been under scrutiny for their systems and practices that promote racism, inequality, and inequity. The field of behavior analysis, with its Eurocentric roots and observed lack of diversity, equity, and inclusion, is not exempt from such evaluations. It is time that we take a look in the mirror and evaluate our own professional, research, educational, and clinical practices, and work towards creating a new, more inclusive, field of behavior analysis that promotes anti-racism and cultural humility.

Keywords: racism, anti-racism, cultural humility, behavior analysis, equity, inclusion 
BEHAVIOR ANALYSIS AND ANTI-RACISM

\section{Editor's Note:}

This manuscript is being published on an expedited basis, as part of a series of emergency publications designed to help practitioners of applied behavior analysis take immediate action to address police brutality and systemic racism. The journal would like to especially thank Jovonnie Esquierdo-Leal and Melody Sylvain for their insightful and expeditious reviews of this manuscript. The views and strategies suggested by the articles in this series do not represent the positions of the Association for Behavior Analysis, International or Springer Nature.

\section{Guest Editor, Denisha Gingles}


BEHAVIOR ANALYSIS AND ANTI-RACISM

\section{A Look in the Mirror: How the Field of Behavior Analysis Can Become Anti-Racist}

Sparked by the most recent murders of Black Americans at the hands of police officers, millions of people flooded streets around the world in protest (Buchanan et al., 2020). This has led to discussions of police brutality, systemic racism, and racial inequalities both within and outside the field of behavior analysis. In recent months, we have seen a number of behavior analysts and behavior analytic organizations acknowledge the need for racial justice and signal their commitment to change with position statements such as Blackout Tuesday and social media posts (Vincent, 2020), to name a few. Despite this, most behavior analysts are likely still not collaborating and utilizing technology available to address racism within the field. Though behavior analysts have long believed that the field could change the world and that a science of human behavior could be utilized to create a society that was socially just and fair (Biglan, 1995, 2015; Levy et al., 2019; Skinner, 1953, 1987), it is also important to reflect on our own practices and evaluate whether we are living up to the values that we promote.

\section{Behavior Analysis, Diversity, and Anti-Racism}

While reviewing the historical context of racism in the United States (U.S.) is beyond the scope of this paper, it is important to understand how racism has and continues to influence systems and structures that oppress BIPOC. Race is commonly defined as a group of people that share certain, often distinctive, physical traits (Blakemore, 2019; Merriam-Webster, n.d.). Race is conceptualized as a social construct, considering that racial categorizations have shifted across the centuries and that there is little genetic variation across human beings of different races (Miller \& Garran, 2017). Despite the constructed nature of race, it does have a very tangible impact, particularly for Black Americans, as it prominently intersects with one's cultural identity. Furthermore, race has often been used to characterize people and suggest differences of 
BEHAVIOR ANALYSIS AND ANTI-RACISM

inherent superiority and humanity (Blakemore, 2019; Matsuda et al., 2020). Racism, a by-

product of racial classification, is the combination of racial prejudice (i.e., discriminations that

are based on assumptions) and power disparity (i.e., authority afforded to a majority group

through social practices and structures; Alberta Civil Liberties Research Centre, n.d.; Oluo,

2018). Racism commonly manifests as social and political exclusion, discrimination, and

violence, as well as one identified group dominating another by way of oppression, slavery, genocide, colonialism, and other forms of mistreatment (Blakemore, 2019; Matsuda et al., 2020;

Miller \& Garran, 2017). Essentially, racism promotes social privilege to some groups (in the U.S., white Americans), while enabling social inequality among others, specifically, in the U.S., BIPOC (Blakemore, 2019).

It is important to draw a distinction between racial equality and racial equity. In this context, the term equality refers to providing equal access to resources. Equity, on the other hand, refers to providing adequate resources necessary to address historic systemic barriers so that BIPOC have equal access to opportunities. For example, in response to the 2020 COVID-19 pandemic, many schools transitioned to remote learning (Center for Disease and Control Prevention, 2020). To assist with this abrupt transition, school districts distributed laptops to students. While providing equal access to laptops was a necessary component of remote learning, there are additional disparities to consider, such as access to the internet (Students of Color Caught in the Homework Gap, n.d.; Valenzuela et al., 2020), an environment conducive to learning (Center for Disease and Control Prevention, 2020; Valenzuela et al., 2020), and parents' ability to facilitate learning (Valenzuela et al., 2020). As a result of these systemic variables, BIPOC students were and continue to be differentially impacted by the COVID-19 pandemic with respect to education (C.S. Mott Children's Hospital, 2020). To properly address inequity, 
BEHAVIOR ANALYSIS AND ANTI-RACISM

one must consider the unique ways that a population is impacted by different contexts in which children are learning, by not only providing resources but eliminating barriers to achieving favorable academic outcomes.

Racism can exist across multiple levels, including individual (i.e., individual attitudes and behavior), systemic (i.e., embedded and perpetuated within an institution or organization via policies and practices of a society or social system), and cultural (i.e., embedded within the values of the dominant culture; Henry, 2004; Miller \& Garran, 2017). As with all human behavior, behaviors that make up racism are taught, imitated, and maintained. This may occur incidentally, or such behavior may be explicitly reinforced via deliberately arranged contingencies. While an examination of the metacontingencies (i.e., contingencies of cultural selection; Glenn, 2004) selected to maintain racist systems is outside of the scope of this paper, they are a product of systemic racism and, therefore, can be used to shape and promote anti-racist behaviors.

Organizations tend to mirror the structural, institutional, and programmatic means of power and privilege of the society in which they exist (Miller \& Garran, 2017). Some organizations take a colorblind approach, acting as if racism and racist structures do not exist unless explicitly set up that way. Others are multicultural, attempting to value all employees, respect their varying cultural background, and make value statements about diversity and marginalized communities without challenging the hierarchies or systems that allow for the existence of institutional racism. Unfortunately, the latter approach often ends up tokenizing BIPOC by hiring them to demonstrate their lip-service commitment to diversity; nonetheless, BIPOC continue to be systematically disadvantaged, as the structures designed to marginalize them remain in place. 
BEHAVIOR ANALYSIS AND ANTI-RACISM

Multicultural organizations tend to focus on cultural competence, which is the idea that the path to addressing racial and cultural divides is to acquire knowledge and awareness about said race or culture. Although cultural competence is valuable, critics have highlighted how the term "cultural competence" is limiting because it implies that learning has an endpoint rather than being continuous (Prasad et al., 2016). An alternative perspective for behavior analysts to take may be that of cultural humility. Though new to behavior analysis, cultural humility has been promoted in the medical, social work, and counseling fields throughout the last several decades (Wright, 2019). Cultural humility may be best thought of as a value that guides one's interactions with individuals of different cultural backgrounds - it is an ongoing pattern of behavior rather than an achievable terminal outcome (Tervalon \& Murray-Garcia, 1998). Cultural humility encourages us to acknowledge that meaningful differences exist across different cultures, as well as to understand that we can never be fully competent in cultures of which we are not members. Thus, to be culturally humble is to engage in not only life-long learning but also consistent critical analysis of one's own biases and prejudices, the sources of those prejudices, and the impact they may have on others (Tervalon \& Murray-Garcia, 1998). An equally important tenant of cultural humility is to recognize and challenge the inherent power imbalances that often exist when working with members of other cultures, with the understanding that the responsibility of those within helping professions extends beyond the immediate therapeutic relationship. To be consistent with a value of cultural humility, one must actively address inequalities and inequities that exist at both an individual and institutional level to fully help clients achieve long-term, meaningful outcomes (Fisher-Borne et al., 2015). In the context of social justice, individuals must engage in ongoing advocacy both within and outside the organizations they are affiliated to in order to contribute towards broader, systemic change. 
BEHAVIOR ANALYSIS AND ANTI-RACISM

Ideally, organizations and individual members of the behavior analysis community need to be decidedly anti-racist; not only demonstrating a commitment to diversity, but also examining the unequal power dynamics within the organization and incorporating cultural humility into their practice (Miller \& Garran, 2017). Anti-racist organizations acknowledge that systemic racism influences their organizational structure and policies. By critically evaluating their structures, anti-racist organizations seek to shift active racist systems to become more equitable and work to create access for those whom the system typically excludes (Liu, 2020).

Many behavior analysts have discussed the lack of inclusion and representation in the field and have highlighted the impact that the lack of racial representation and cultural humility may have on behavior analytic research and practice (Conners, 2020a; Covenant 15:16, 2019; Dennison et al., 2019; Fong et al., 2017; Jackson, 1976; Meadows, n.d.; Szabo, 2020; Wilhite, 2015). This observed lack of diversity, equity, and inclusion is often indicative of systemic racism and reflective of the current systems and policies in place. It is not surprising that behavior analysis, which evolved from Eurocentric models, needs to be adapted to reflect the current cultural variability of both its members and the individuals we serve (Jackson, 1976). Mainstream social services and organizations (e.g., hospitals, mental health agencies, college counseling services, etc.) that evolved from a Eurocentric model were not originally established to provide services to BIPOC. As such, they often provide services without consideration of cultural differences and maintain status quo with leadership positions that are not representative of the communities they serve (Miller \& Garran, 2017).

Given these ideas, we argue that embracing cultural humility and adopting anti-racist practices are important steps in addressing systemic racism within the field of behavior analysis (Wright, 2019), which will ultimately result in higher quality services for those we work with. 
BEHAVIOR ANALYSIS AND ANTI-RACISM

This paper discusses racism at various levels, including professional organization and governing bodies, ethics, education and training, behavior analytic organizations, and individual practitioners. Specifically, actionable steps related to cultural humility and adopting anti-racist practices are provided in effort to create a more inclusive and representative field of behavior analysis.

\section{Professional Organizations/Governing Bodies}

Governing bodies and professional organizations have a large influence over the behavior of their members, dictating not only the skills, values, and repertoires considered necessary to fulfill the requirements of the profession, but also controlling the contingencies that maintain such behavior. Therefore, it is imperative that professional organizations take an active role in terms of governing, disseminating, and modeling how to address racism, including how to make anti-racist behaviors applicable across various contexts in the field. With that in mind, we encourage the major behavior analytic organizations at both the state and national levels to transition from a purely multicultural focus to one that is anti-racist.

Some steps have already been made in this direction. The Association for Behavior Analysis International (ABAI) and the Association of Professional Behavior Analysts' (APBA) websites have both included links on their homepage to allow practitioners to connect with committees dedicated to diversity, equity, and inclusion (DEI). Additionally, continuing education (CE) opportunities are available on both organizations' webpages, including APBA's live webinar regarding behavior analysis and racism (APBA, 2020) and ABAI's CulturoBehavior Science for a Better World virtual conference (ABAI, 2020). Along the same lines, the Behavior Analytic Certification Board (BACB) posted a statement about their commitment to DEI on Facebook in the weeks following George Floyd's murder by a Minneapolis police 
BEHAVIOR ANALYSIS AND ANTI-RACISM

officer. Recently, in an attempt to collect demographic information of its members, the BACB updated their certificate portal and requested members to update information related to gender, race, and ethnicity. Specific to gender, members were asked to select one of the following options: male, female, non-binary, prefer not to answer, or other. Specific to race and ethnicity, members were asked to select one of the following options: American Indian/Alaska Native, Asian, Black, Hispanic/Lantinx, Native Hawaiian/Other Pacific Islander, white, or prefer not to answer. While, at first glance, this appears to be a step in the right direction, the data collection system was notably flawed. The questionnaire only allows members to select one option, which may lead to identity erasure of multi-racial or multi-ethnic certificants in addition to conflating race and ethnicity. At the time of submission, these concerns had not yet been addressed or rectified.

While these efforts are important, there is still much more that needs to be done for the aforementioned organizations to become substantively anti-racist. As discussed above, anti-racist organizations take an active role in examining and modifying structures that maintain racial inequality. By articulating policies, making resources readily available to members, and encouraging continuous learning within the scope of anti-racism as an established core value of their organizations, they begin to address racial inequities. Additionally, they recognize that racism creates disproportionate opportunities and penalizes BIPOC in both obvious and subtle ways, and they work on manipulating existing systems to create more access for the impacted group. It would behoove behavior analytic organizations and governing bodies to examine their own anti-racist practices and reference the practices of similar organizations in related fields.

As an example, the National Association of Social Workers (NASW) have included a statement emphasizing their commitment to social justice that is centrally located on their 
BEHAVIOR ANALYSIS AND ANTI-RACISM

website and resources such as trainings, critical conversations (e.g., Congressional briefings), and additional articles discussing social work and police reform (NASW, 2020).

Another area of concern is the lack of representation within our professional

organizations. Evidence reported by the BACB and lived experience reported by many behavior analysts of color suggest that the field continues to be predominately white, middle-class, English-speaking individuals, with an especially noteworthy lack of Black members (BACB, n.d.; Covenant 15:16, 2019; Wilhite, 2015). In addition, at the time of publication, there is little racial diversity among the leadership and boards of professional organizations. This lack of representation poses at least two major issues that will (and arguably already has) hinder the effectiveness of the field to fulfill its mission. First, a lack of representation will only further deter BIPOC from pursuing careers in behavior analysis (Estrada et al., 2016; Flores et al., 2019). Second, the dearth of minority voices will likely result in important cultural variables being ignored or absent from crucial policy decisions (Gourdine \& Algood, 2014). Such oversight may not necessarily be intentional, as individuals are not always aware of the knowledge that they lack.

In the Facebook post by the BACB (2020), they stated:

The BACB is committed to diversity and equality and places the highest value on inclusivity in all environments, including professional settings, universities, and the community at large. Although the BACB, as the profession's certification board, cannot speak for or represent our discipline as a whole, our professional associations can and do. (para. 2)

Within the framework of anti-racism, a commitment to diversity and equity would, at minimum, have organizations share resources, adapt their systems to ensure both the representation and 
BEHAVIOR ANALYSIS AND ANTI-RACISM

safety of BIPOC at various levels of the organization, and promote a continuous evaluation of

their systems and practices to ensure accessibility for BIPOC and accountability for white people.

\section{Ethics Codes}

As in most helping professions, the field of behavior analysis has a set of ethical rules and guidelines which codifies behavior analysts' responsibility with respect to a multitude of applied practice and research domains and provides contingencies for adhering (or not adhering) to these codes of conduct. The current set of guidelines, referred to as the Professional and Ethical Compliance Code for Behavior Analysts, has been in effect since 2016 (BACB, 2014). Despite its importance as a guiding force, the current compliance code specifies very little in the way of cultural and racial awareness, and next to nothing on diversity, equity, and inclusion. Perhaps the most applicable are Code $1.05(\mathrm{c})$, which gives limited guidance on how a behavior analyst should respond to situations where differences, including race, cultural, and ethnicity, impact their work, and Code 7.01, which affirms behavior analysts' responsibility to promote an ethical culture, also lends direction to these topics (BACB, 2014). Concerningly, the lack of mention of racism, diversity, equity, and inclusion has had the effect (unintended or not) of communicating that such topics are unimportant or less deserving of attention than others.

In recognition of the importance of cultural awareness and DEI (and likely in response to the calls for change from many behavior analysts), in December 2020, the BACB released a new set of ethical guidelines, which will officially come into effect in January 2022. This new version, referred to as the Ethics Code for Behavior Analysts, includes ethical codes related to cultural responsiveness and diversity (Code 1.07), nondiscrimination (Code 1.08), awareness of personal biases and challenges (Code 1.10), and incorporating and addressing diversity (4.07; 
BEHAVIOR ANALYSIS AND ANTI-RACISM

BACB, 2020). These additions bring awareness to areas in which behavior analysts may witness and/or engage in behaviors related to discrimination and cultural biases within the scope of their practice and/or supervision.

Despite these notable additions, the new ethics code offers little tangible guidance for how these guidelines should be used regarding racism. The language included in these codes still largely leaves it up to individual practitioners, who, as previously stated, are mostly white, to evaluate the extent of their biases and degree of cultural responsiveness. Furthermore, the new ethics code does not include mention of behavior analysts' responsibility to address or dismantle the existing mechanisms perpetuating the inequity within our field. We, and others, argue that because these are paramount ethical concerns, any code of ethics is incomplete without guidelines pertaining to these topics. In the same way, there are codes for promoting an ethical culture within behavior analysis; codes are needed for promoting an anti-racist culture as well.

We can look to our neighboring fields as models for how to include a code of ethics that truly reflects dignity and respect in all aspects. The American Counseling Association, the National Association of School Psychologists, the American School Counselor Association, and the National Association of Social Works all have numerous items within their official ethical compliance documents that address the role of cultural variables in service delivery, provide guidelines for navigating and responding to discrimination in all forms, and stress the importance of social justice advocacy. In addition, these ethical codes are distributed across multiple sections of the documents, thus further demonstrating their relevance to all aspects of the profession. The biggest advantage of these documents, however, is that they are explicit in communicating their field's values and perspectives on this topic. While many of the BACB's ethical compliance codes may be interpreted through an anti-racist lens, their lack of specificity makes it possible to 
BEHAVIOR ANALYSIS AND ANTI-RACISM

behave in a manner that is inconsistent with anti-racism, yet, those behaviors would still be considered ethical. Likewise, adhering to these codes can be especially difficult when demographic variables and information on the impact of interventions is not readily available in the literature to guide clinicians, supervision, and/or training (Jones et al., 2020).

Including a BACB's ethical code of this nature could aid organizations and individuals in being stricter and increasingly aware of microaggressions that occur in the workplace and/or towards colleagues and/or clients. Microaggressions are defined as "everyday derogations, slights, and invalidations that are often delivered to people of minority or marginalized backgrounds" (Lui \& Quezada, 2019, para. 1). Finally, the NASW's (2017) ethical codes have specific codes on ethical responsibilities to society, as well as codes related to social and political action. While the entire code is a useful model, it is notable that section b of code 6.04 states, "Social workers should act to expand choice and opportunity for all people, with special regard for vulnerable, disadvantaged, oppressed, and exploited people and groups" (NASW, 2017, p. 19). Ethical codes regarding society, politics, and the field as a whole are necessary to guide new and current practitioners in fostering socially significant changes in our clients' lives as well as in our respective workplace.

The responses from Sellers et al. (2020) to Rosenberg and Schwartz's (2019) criticisms of several sections of the BACB's ethical codes should also be noted. Sellers et al. (2020) reminded the reader of the following ethical code: "provides a framework for practitioners and consumers to evaluate a practitioner's behavior to (a) gain an understanding about questionable behavior, (b) help identify more ethical courses of action, and (c) determine when to report potentially unethical behavior" (p. 2). The above recommendation would be relevant and needed to evaluate practitioner and organizational behavior. Sellers et al. (2020) also noted that, in 
BEHAVIOR ANALYSIS AND ANTI-RACISM

addition to the ethical codes, our behavior may also be shaped by engaging with other

practitioners, attending conferences, and acquiring $\mathrm{CE}$ units for recertification. It will be urged in this paper that the ethical codes, recertification process, and CE requirements be altered moving forward. Lastly, Sellers et al. (2020) reminded the reader that there are subject matter experts available and novel information regarding practices of other fields that can be included when discussing the standards of behavior analysis. While there are potential barriers to making these updates to the codes (e.g., slow-moving accredited processes for updating codes), it is hoped that the ethical codes identified above can provide initial content discussions when enhancing antiracist and non-discriminatory codes.

\section{Actionable Steps}

1. Actively promote and display anti-racist values across multiple platforms, including websites and social media:

2. Provide access to resources on anti-racism, highlight research articles from other fields on topics of diversity, equity, and inclusion, and post links to CE resources on such topics directly on the organizations' websites.

3. Update CE requirements and standards for maintaining board certified behavior analyst (BCBA) certification to not only include ethics and supervision but also diversity, equity, and inclusion; this is not uncommon for state licensure in other fields (e.g., Ohio Psychological Association; Beautiful Humans Change, 2020).

4. Update the ABAI-verified course sequence and BACB's task list to include topics related to cultural humility and cultural responsiveness (Beautiful Humans Change, 2020). 
BEHAVIOR ANALYSIS AND ANTI-RACISM

5. Collect and report data on members' demographic variables to improve independent evaluation of cultural diversity within the field (Beautiful Humans Change, 2020; Conners, 2020a).

6. Create a task force (e.g., diversity, equity, and inclusion task force) at organizations.

7. Actively solicit voices of Black authors and presenters at conferences and workshops, in podcasts, and in other prominent materials (e.g., textbooks).

8. Release regular public documents outlining the "state of the organization," with particular emphasis on diversity, equity, and inclusion initiatives. This should include statements of the specific goals set forth by the organization, as well as a report of progress made towards those goals and a description of modification to be made if progress is not being made.

9. The BACB should add ethical codes that explicitly address anti-racism and discrimination.

10. The BACB's ethical codes should be updated to provide more direction for behavior analytic researchers on the expectations of reporting demographic variables (e.g., ethnicity, setting) in research studies.

11. The BACB should solicit and remunerate a wider range of diverse subject matter experts to provide suggestions and make changes for behavior analysis and practitioners (Sellers et al., 2020).

12. The BACB should work towards increasing the transparency of how ethical codes are determined. 
BEHAVIOR ANALYSIS AND ANTI-RACISM

13. The BACB should utilize existing mechanisms to intentionally solicit feedback and/or suggestions related to the improvement and addition of new ethics codes, especially from more culturally and racially diverse populations.

14. The BACB should allocate further resources to evaluate their current ethics codes more frequently to ensure they are relevant, applicable, and congruent with current social values and cultural changes.

\section{Education}

Despite some advances, many have noted a continued lack of diversity across disciplines in higher education (Estrada et al., 2016; Flores et al., 2019; Griffin \& Muñiz, 2011; Vela et al., 2010). The U.S. Department of Education (2016) has stated that, "During the past 50 years, the U.S. has seen racial and ethnic disparities in higher education enrollment and attainment, as well as gaps in earnings, employment, and other related outcomes for communities of color” (p. 1). To add to this, Leonard et al. (2002) mentioned that even with efforts to remediate the issues at hand, faculty diversity and inclusion across colleges/universities has not yet been reached, especially in more senior positions. Chen (2017) echoed similar sentiments when they discussed the inadequate representation of ethnic minorities in student and professional bodies. Data collected by the Integrated Postsecondary Education Data System suggested that the number of BIPOC students who have earned degrees in applied behavior analysis (ABA) has steadily increased between 2012 and 2017 (Data USA, n.d.). Nonetheless, data summarized for 2017 highlighted that approximately $60 \%$ of all degrees awarded were to individuals who are white, approximately $12 \%$ to individuals who are Hispanic or Latinx, and approximately $11 \%$ to individuals who are Black (Data USA, n.d.). 
BEHAVIOR ANALYSIS AND ANTI-RACISM

There are many barriers that BIPOC may experience in relation to access to education.

These include but are not limited to implicit attitudes (e.g., feedback, consequences; Perception Institute, 2014; Tesfaye, n.d.); lack of opportunities to participate in school programs (e.g., science, technology, engineering, and mathematics; Sablich, 2016); and restrictive access to funding resources (Tesfaye, n.d.), mental health support (Tesfaye, n.d.), and well-funded elementary, middle, and high schools and colleges (Edbuild, n.d.; Metivier, 2020; Sablich, 2016). Language is an example of another barrier that BIPOC may experience in higher education in the U.S. As a result of a Western ethnocentric system, some professors and higher education staff members attempt to modify students' writing and communication styles. This may include tone policing, which is a form of diversion, where the focus is placed on the delivery of the message, as opposed to the meaning. Tone policing expects students to modify their own verbal behavior to conform to their professors' learning history and communication style (Martin, 2018). This may also lead to code-switching, where an individual who speaks multiple dialects or languages will switch from one to the other depending on context to enhance social meaning. Often, this means that students are expected to conform to speaking in a dialect that is labeled "professional" by their faculty but can be traced to the construct of white supremacy (American Psychological Association, n.d.). Though people who engage in these behaviors may not have ill intentions, it remains a form of microaggression, which, as many authors have stated, “... are extremely invalidating to marginalized students" (as cited in Kang \& Garran, 2018, p. 5).

Other important aspects of behavior analysis that are directly impacted by systemic racism are case conceptualizations and examples that students are exposed to and used for class discussions. As students and professionals in the field of behavior analysis, we seem to be promoting a predominately Western and ethnocentric conceptualization of behavior and 
BEHAVIOR ANALYSIS AND ANTI-RACISM

behavior-analytic systems that may not be consistent with other cultures. For example, the vignettes and exemplars provided in many of the common behavior analytic textbooks often focus on stimuli prompts that are common in Western societies and/or in predominately white, English-speaking communities, and commonly suggest a certain universality to such stimuli that may not be functional or necessary in non-Western societies. Too often, students learning the application of behavior analysis are taught to help clients be successful in the practitioner's natural environment rather than the culturally-specific natural environment of the client. This poses a potentially huge problem, as many of us work with diverse populations and in different countries, though the field overall continues to be largely North American, white, Englishspeaking, and middle- to upper-middle class.

Higher education in the field of behavior analysis, which is embedded within the larger higher education system, is not immune to such concerns. Given the above factors, it should not be surprising that courses related to diversity, equity, and inclusion, in which issues related to racism and cultural humility are typically discussed, are not required in all undergraduate and graduate ABA programs (e.g., as cited in Conners, 2020b; Dennison et al., 2019). For example, Conners (2020b) evaluated diversity training amongst behavior analysts and concluded that $89.47 \%$ of participants reported that the current curriculum where they taught did not include a course on diversity, and only $23.68 \%$ of participants thought that their respective programs were thoroughly equipped to teach diversity. Given the absence of required diversity-related coursework, it is clear that changes need to be implemented to ensure that future researchers, academicians, and practitioners, to name a few, are not only exposed to concepts such as diversity, equity, inclusion, and cultural humility in their coursework but also demonstrate it through applied practice. 
BEHAVIOR ANALYSIS AND ANTI-RACISM

\section{Research and Publication}

It has been noted that behavior analytic literature often fails to sufficiently report demographic variables, including variables related to race and culturally diverse populations and settings (Dennison et al., 2019; Jones et al., 2020). This includes information regarding research participants' ethnicities as well as home languages (Dennison et al., 2019; Li et al., 2017). Given that research informs practice, the failure to report culturally specific variables may lead to a lack of understanding about the potential effectiveness of measurement or assessment instruments (Dennison et al., 2019). Jones et al. (2020) examined the Journal of Applied Behavior Analysis between the years of 2013 and 2019 to determine how many authors reported participants' demographic information in their studies. These demographics included ethnicity/race, socioeconomic status, gender, sex, and age. Some of the most staggering results described by Jones et al. (2020) were that $2 \%$ of articles during these years included socioeconomic status, and only $7 \%$ reported race. In addition, results from Jones et al. (2020) indicated that of the articles that reported relevant demographic variables, 69\% of participants were Caucasian, 20\% were African American, and less than 5\% were Hispanic, Asian, Biracial, Native American, or “Other." Jones et al. (2020) also reported that only four languages were identified as a studied demographic variable from 2013 to 2019, which included English, Portuguese, Georgian, and Spanish, with the vast majority reporting English-speaking participants.

The researchers asserted that reporting more demographic variables may not only assist in identifying functional relations between treatment results, but also in promoting future research to use these variables "... functionally rather than structurally" (Jones et al., 2020, p. 8). The evaluation of whether goals, procedures, and effects are socially valid from members of our field, consumers, and those from other fields could also be enhanced if behavior analysts are 
BEHAVIOR ANALYSIS AND ANTI-RACISM

ensuring that Black and other culturally diverse individuals are being considered for reporting, assessment, and treatment (Jones et al., 2020; Kunze et al., 2019).

\section{Actionable Steps}

1. Include permanent, anti-racism statements on website(s) and any other platform(s) that is(are) used to disseminate information to students and faculty.

2. Organizations should create and implement proactive and reactive policies and have a designated department (e.g., multi-cultural office) or individual (e.g., ombudsman) to address concerns with, outline the procedures that an educational institution will take to address concerns, advocate within the institution, and ensure that all concerned parties are informed and trained accordingly.

3. Create a school-wide team composed of members from each department or if possible, a group of individuals in each department, to address issues related to microaggression, tone policing, and racism.

4. Assign a specific individual or group of people to review and ensure that DEI is an integral part of each course offered (Chen, 2017).

a. An example of a vignette that could be utilized by instructors and potential ways to address cultural humility are provided in Appendix A.

b. Universities and institutions should actively work to ensure that this responsibility does not fall on the BIPOC members of their faculty. Rather, they can demonstrate their commitment to DEI by ensuring that all faculty members are equipped to incorporate DEI into their courses.

5. Make a deliberate attempt to hire and recruit individuals who embody diversity (Chen, 2017). 
BEHAVIOR ANALYSIS AND ANTI-RACISM

6. Implement mandatory training that will expose staff members to cultural humility and diversity advocacy (Chen, 2017; Wright, 2019).

7. Offer sufficient resources (e.g., grants/scholarships) and services (e.g., facilities specifically dedicated to handling diversity) to promote and uphold a diverse body of students and professionals (Chen, 2017).

8. Ensure that several mentors with diverse backgrounds are available to BIPOC (Flores et al., 2019; Valentine, 2015).

9. Work towards decreasing tuition, increasing grant and scholarship opportunities, ensuring applications for funding opportunities are fair and equitable, and removing limits to student loan amounts, to name a few.

10. Other recommendations by Valentine (2015) include:

a. Inviting current students to pursue higher education.

b. When reviewing students' applications, assess “.... cognitive and noncognitive abilities, such as communication and critical thinking skills that may better predict success among students of color" (para. 3). This thought was echoed by Kendi (2016) who highlighted that standardized testing puts certain racial groups at an initial disadvantage.

11. Institutional review boards and editorial procedures can require researchers to describe the participants being recruited ( $\mathrm{Li}$ et al., 2017).

12. Journal policies should be altered to encourage researchers to report demographic variables when submitting manuscripts (Jones et al., 2020). 
BEHAVIOR ANALYSIS AND ANTI-RACISM

13. Consistent with the suggestions of Jones et al. (2020), Brodhead et al. (2014), Li et al. (2017), and Severini et al. (2018), behavior analytic research should include participant demographic and setting information.

14. Consistent with recommendations made by McSweeney et al. (2000) and Li et al. (2018), research should be conducted to examine and report on how many culturally diverse authors as well as editors are participating in behavior analytic journals.

a. For example, a study conducted by McSweeney et al. (2000) and later followed up by Li et al. (2018) analyzed article authorship as well as editorial board membership for women in the field. Similar studies should be extended to Black and culturally diverse behavior analysts to ensure well-rounded perspectives.

15. Consistent with Dennison et al. (2019), researchers should examine how the "language and cultural match" between a researcher and participant could impact communication, appropriateness of recommendations, the consent process, and goals (p. 888).

\section{ABA Organizations and Providers}

Systemic racism may also be present amongst ABA organizations and providers. This may be evident in their policies and procedures, and with the lack of representation amongst practicing clinicians, including both the supervisory teams and registered behavior technicians (RBTs). Some of the areas in which organizations fail to account for systemic racism include the lack of policies in place to ensure the safety of marginalized employees against racist behavior and microaggressions. For example, when faced with a situation where a BIPOC employee experiences racism and/or microaggressions with a client, some organizations may prioritize revenue over the safety of their BIPOC employees by either requiring that the BIPOC employee "ignores" it or by reassigning the said employee(s) to a different client. Additionally, many 
BEHAVIOR ANALYSIS AND ANTI-RACISM

organizations do not provide a space for these employees to report such interactions with families of clients or fellow employees, nor do they enact protocols to periodically evaluate the behavior of their employees to facilitate growth in inclusive behaviors and reduce biased ones. Employees from marginalized backgrounds may lack access to mentorship opportunities from others with whom they share cultural identities. All of these systemic failures to support BIPOC employees can contribute to job anxiety and burnout on a professional level; acute as well as chronic stress and an increased risk of mental health disorders on a personal level may also be experienced and reported (Williams, 2018).

Lastly, organizations may have policies related to behavior support and crisis intervention that involve including emergency responders, such as the police. However, police are often not trained to support neurodivergent and/or disabled individuals safely and without the use of additional, often unnecessary, force. Furthermore, the decision to call the police is often made without consulting with the individual client and/or their family in advance. While it may be common practice for an organization to involve law enforcement when they feel that they are not able to effectively support a client in crisis as a way to protect their staff and other individuals in the community, this practice may not be in the best interest of the client, especially if they are BIPOC (Abrams, 2020; Disabled Word, 2013; Kineavy, 2018). Alternatives to calling the police have become more prevalent in recent years. For example, Mental Health America (MHA; n.d.) provides information about and links to alternative options to calling the police or 911; the list of resources is broken down by major cities. Likewise, Black Emotional and Mental Health Collective (BEAM; n.d.) provides a list of mobile crisis unit services that are available within in the U.S. Similarly, there have recently been pilot projects in both Ottawa (Canada) and New York (U.S.) that have initiated mental health response teams to work with the police to support 
BEHAVIOR ANALYSIS AND ANTI-RACISM

those in crisis who may need emergency support (Samuel, 2020; Vandermeer, 2020; Williams \& Sgueglia, 2020).

\section{Actionable steps}

1. Implement anti-racism policies and create proactive and reactive procedures to address racism and microaggressions within your organization, including those that an employee may experience from clients and other staff members.

2. Organizations should explicitly state their stance on racism as well as their diversity, equity, and inclusion policies when hiring employees and accepting new clients.

a. This can include providing demographic data of the racial makeup of the organization.

b. Organizations should establish anti-racism as a core value (i.e., the steps the organization is taking to combat racism). This demonstrates clear behavioral expectations and ensures a safe space for both employees and clients.

3. Designate specific departments to address issues that may impact marginalized employees and clients.

4. Create a space for employees to address racist experiences and racial trauma.

5. Incorporate clear policies related to practitioner-client communication channels either via a translator or a consenting practitioner who speaks the language.

6. Include policies to ensure that practitioners are asked to engage in self-reflection, assess their own biases towards specific communities, and make the necessary changes before they are asked to work with those communities. 
BEHAVIOR ANALYSIS AND ANTI-RACISM

7. Ensure that the organization has a pool of diverse staff available for clients, such that practitioners that are members of a specific community or cultural group are available upon a client's requests, with the practitioner's consent.

8. Incorporate continual cultural humility training that includes the use of performance criteria (e.g., Central Vancouver Island Multicultural Society, n.d.) and/or cultural competency checklists (e.g., Goode, 2009) to continually evaluate one's current skill set and identify areas of growth. Training could also include very clear standards for diversity, equity, and inclusion across several of the above avenues.

9. Ensure that staff are trained using a wide variety of cultural exemplars of clients they may be working with and cultural-specific contexts they might be exposed to.

10. Ascertain that the client and their family are involved in the creation of behavior support plans (BSPs) and other crisis intervention policies to ensure that assent and consent are obtained before escalated measures are employed.

a. Caregivers and clients should also be reminded that they are entitled to modify or remove consent and/or assent at all times, even on previously agreed upon service plans or BSPs.

11. Consider including alternatives to calling law enforcement during crisis intervention, such as those recommended by MHA (n.d.) and BEAM (n.d.).

\section{Individual Practitioners, Researchers, and Academicians}

Concerns related to racism, diversity, equity, and inclusion at the practitioner level are especially evident when one considers the potential effects of having practitioners who lack training in cultural humility. Unfortunately, these individuals are more likely to engage in microaggressions and be complicit in white supremacy. For example, many of our assessments 
BEHAVIOR ANALYSIS AND ANTI-RACISM

have been created using white/Western culture specific variables and standards. This is evident in the examples provided, the questions or prompts suggested, and/or the materials that are provided for conducting assessments. It should be noted that available assessments, as well as associated stimuli, are commonly supplied to the behavior analyst through their employer or organization. Likewise, many translations or adaptations of assessments are completed by individual practitioners and are therefore not standardized. Concerns include but are not be limited to: 1) using teaching exemplars that do not include the client's primary language and/or culturally relevant variables; 2) using assessments that do not reflect a client's primary language or individual learning history, which, in turn, could lead to incorrect evaluations of their skills due to their proficiency (or lack thereof) in English; (3) one's inability to develop rapport with parents and guardians if they are not trained to be sensitive to culture-specific social behaviors; and (4) selecting social reinforcers that may not be culturally acceptable. This may impact a behavior analyst's ability to provide quality applied behavior analysis services as well as design and conduct research effectively if they are not trained to factor in cultural variables in their clinical practice (Dennison et al., 2019; Jackson, 1976; Jones et al., 2020; Meadows, n.d.; Wilhite, 2015).

Dennison et al. (2019) discussed several potential barriers that practitioners may face when working with racially marginalized populations. While, in theory, these concerns should fall under the realm of social validity, in practice, they are often ignored (Levy, 2019; Mittelman, 2019; Sriman \& Klaptach Totsch, 2019). Jackson (1976) discussed potential barriers (e.g., the use of colonial perspectives and a biomedical approach to race, behavior modification goals under the control and selection of the majority of the population) that the field of behavior analysis may face when working with Black clients, especially since the field defaults to 
BEHAVIOR ANALYSIS AND ANTI-RACISM

Eurocentric approaches to tackle mental health. It is imperative for behavior analysts to discuss how other cultures approach mental health diagnoses to ensure that our analyses and services are consistent with the client's cultural practices and individual learning histories (Jackson, 1976).

Dennison et al. (2019) detailed the barriers that practitioners face when working with culturally and linguistically diverse families, including a lack of diversity among researchers and practitioners, the presence of attitudes and biases with respect to specific cultures, a disconnect between languages spoken by clients, clinicians, and therapists, a lack of understanding of the impact of socio-cultural and socio-economic stressors, multilingualism, and more.

Individual practitioners may also engage in racism and/or microaggressions through the programs that are implemented by using stimuli that do not represent the individual client (e.g., excluding examples of persons who share similar physical traits or culturally relevant clothing) and training relations with stimuli that are inconsistent with an individual's cultural context. This further emphasizes the importance of including the individual client and/or their family when creating and implementing program goals, as well as selecting stimuli that will be used in programming. For example, when training concepts such as community helpers, practitioners may train the client to perceive a police officer as a source of help and safety, which may be inconsistent with their reality and cultural context and may lead to them being in a vulnerable or risky situation outside of a therapeutic setting. Engaging in practices consistent with anti-racism and cultural humility will enable the practitioner to evaluate the social significance of the conceptualizations and relations being trained, stimuli and materials being used for assessments and training, and how to respectively and effectively work with the client and their family. 
BEHAVIOR ANALYSIS AND ANTI-RACISM

1. Practitioners should evaluate their own cultural humility and ability to work with a client whose culture may differ from their own.

a. Practitioners should engage in self-reflection and assessment of their current skill set. This could be accomplished through a set of self-reflective assessments specific to cultural humility that could be adapted from assessments, such as those provided by Goode (1989), Leland and Stockwell (2019), or Vendor Resource Management University (2016), to name a few.

2. BCBAs should practice referring clients to practitioners more suited to their cultural identity if they recognize that they may not be well-suited to provide services for said clients while still simultaneously advocating for diversity in the field.

3. BCBAs should engage in self-reflection (e.g., using some of the assessments listed above) to evaluate whether they are a suitable match, in terms of providing support to their supervisees. If the potential supervisor is not deemed a suitable match, they should complete additional training, seek out supervision for themselves, or, if necessary, refer out to a more suitable practitioner.

4. Practitioners should ensure that assessments are individualized to the client's language, culture, and learning history (Fong et al., 2016).

a. For example, using culturally relevant stimuli or verbal behavior to measure a client's competence as opposed to the suggested lists in the Assessment of Basic Language and Learning Skills - Revised (ABLLS-R) appendix.

5. Practitioners should be mindful of multilingual families and ensure that verbal behavior is shaped under multiple environments (e.g., speak English at school, Tamil at home, etc.) or teach verbal behavior across multiple languages. 
BEHAVIOR ANALYSIS AND ANTI-RACISM

6. Practitioners should take extra care when interviewing clients, parents, and other caregivers when creating programs and BSPs. This will ensure that appropriate relations are trained within their individual cultural context.

a. For example, some cultures do not prefer calling the police when faced with an emergency situation; as such, a series of steps that not only reflect cultural practices, but also work towards ensuring that the safety and wellbeing of the client is maintained should be outlined.

7. Specific to individual behavior that may impact governing bodies, individuals and organizations should sign and share petitions and stay informed of the current/future leadership and vote.

a. Individuals should nominate people who lead and promote anti-racist policies in organizations.

8. Current non-Black behavior analysts should participate in conferences and attend presentations led by Black behavior analysts.

a. For example, one could register for and attend the diversity training/presentations at $\mathrm{ABAI}$ and/or the Black Applied Behavior Analysts conference in 2021 (Black Applied Behavior Analysts, Inc., n.d.).

9. Support (financially and / or socially) existing organizations within the field that take active anti-racists stances, such as Black Applied Behavior Analysts, Inc., The ABA Taskforce to Eradicate Social Injustice, Beautiful Humans, etc.

10. Specific to individual behavior that may impact research and journal practices, request an increase in special issues on diversity and the amplification of Black and other culturally diverse authors that are submitting for publication. 
BEHAVIOR ANALYSIS AND ANTI-RACISM

11. Individuals should call out the racist behavior of others within the field, especially when they are systemically advantaged to do so in a manner that their BIPOC colleagues are not.

\section{Conclusion}

While behavior analysis has seen an increase in emphasis on multiculturalism and cultural competence, the field has a long way to go towards fully incorporating cultural humility and anti-racist values. Many of the ideas and concepts mentioned in this paper are outside of the scope and confines of this submission, but we hope that readers will use the references provided to expand their understanding (see Appendix B). We hope that this paper works as a starting point; a call to action for the field to further research, evaluate, and address concerns related to racism, diversity, equity, and inclusion within the field of behavior analysis. It is time that we hold ourselves to a higher standard and create a practice of behavior analysis that is rooted in anti-racism, involve the teaching and practice of cultural humility, and provide objective and measurable systems to ensure that its members are provided with the skills needed to work with an individual who may have a different cultural, ethnic, or religious background than theirs. We hope that this paper inspires the field to move forward from some of our archaic practices and to work towards creating a more inclusive and representative field of behavior analysis. 
BEHAVIOR ANALYSIS AND ANTI-RACISM

\section{References}

Abrams, A. (2020, June 25). Black, disabled and at risk: The overlooked problem of police violence against Americans with disabilities. Time. https://time.com/5857438/policeviolence-black-disabled/

Alberta Civil Liberties Research Centre. (n.d.). What is racism? http://www.aclrc.com/racism$\underline{\text { and-power }}$

American Psychological Association. (n.d.). APA dictionary of psychology. https://dictionary. apa.org/code-switching

Association for Behavior Analysis International. (2020). Home of the science and the practice of behavior analysis. https://www.abainternational.org/welcome.aspx

Association of Professional Behavior Analysts. (2020). Live webinars. https://www.apbahome. $\underline{\text { net/page/live-webinars }}$

Beautiful Humans Change. (2020). Welcome to beautiful humans change. https://www.beautiful humanschange.com

Behavior Analyst Certification Board. (n.d.). BACB certificant data.

https://www.bacb.com/BACB-certificant-datahttps://www.bacb.com/ BACB-certificantdata.

Behavior Analyst Certification Board. (2014). Professional and ethical compliance code for behavior analysts. https://www.bacb.com/wp-content/uploads/2020/05/BACBCompliance-Code-english_190318.pdf

Behavior Analyst Certification Board. (2020). Ethics code for behavior analysts. https://www.bacb.com/wp-content/uploads/2020/11/Ethics-Code-for-Behavior-Analysts2102010.pdf 
BEHAVIOR ANALYSIS AND ANTI-RACISM

Behavior Analyst Certification Board. (2020, June 4). The tragic events over the past week and ongoing social injustices in the US are a stark reminder of the [Status update]. Facebook. https://www.facebook.com/BehaviorAnalystCertificationBoard/posts/3130978453635489

Biglan, A. (1995). Changing cultural practices: A contextualist framework for intervention research. Context Press.

Biglan, A. (2015). The nurture effect: How the science of human behavior can improve our lives and our world. New Harbinger Publications.

Black Applied Behavior Analysts, Inc. (n.d.). About us. https://www.babainfo.org

Black Emotional and Mental Health Collective. (n.d.). Mobile crisis unit services in the United States. https://www.beam.community/mobilecrisis

Blakemore, E. (2019, February 22). Race and ethnicity: How are they different? National Geographic. https://www.nationalgeographic.com/culture/topics/reference/race$\underline{\text { ethnicity/\#close }}$

Brodhead, M. T., Duràn, L., \& Bloom, S. E. (2014). Cultural and linguistic diversity in recent verbal behavior research on individuals with disabilities: A review and implications for research and practice. The Analysis of Verbal Behavior, 30(1), 75-86. https://doi.org/ 10. $1007 / \mathrm{s} 40616-014-0009-8$

Buchanan, L., Bui, Q., \& Patel, J. K. (2020, June 3). Black lives matter may be the largest movements in U.S. history. The New York Times. https://www.nytimes.com/interactive/ 2020/07/03/us/george-floyd-protests-crowd-size.html

Center for Disease and Control Prevention. (2020, July 24). Health equity considerations and racial and ethnic minority groups. https://www.cdc.gov/coronavirus/2019ncov/community/health-equity/race- 
BEHAVIOR ANALYSIS AND ANTI-RACISM

ethnicity.html?CDC_AA_refVal=https $\% 3 \mathrm{~A} \% 2 \mathrm{~F} \% 2 \mathrm{Fwww} . c d c . g o v \% 2 \mathrm{Fcoronavirus} \% 2 \mathrm{~F} 2$ 019-ncov\%2Fneed-extra-precautions\%2Fracial-ethnic-minorities.html

Central Vancouver Island Multicultural Society. (n.d.). Cultural competence self-assessment checklist. http://www.coloradoedinitiative.org/wp-content/uploads/2015/10/culturalcompetence-self-assessment-checklist.pdf

Chen, A. (2017). Addressing diversity on college campuses: Changing expectations and practices in instructional leadership. Higher Education Studies, 7(2), 17-22. https://doi.org/10.55 $\underline{39 / h e s . v 7 n 2 p 17}$

Cochran, G. (2018, June 22). Guest post: The problem with diversity, inclusion, and equity. The Scholarly Kitchen. https://scholarlykitchen.sspnet.org/2018/06/22/problem-diversityinclusion-equity/

Conners, B. M. (2020a). Where is the diversity in applied behavior analysis? https://www.brian connersbcba.com/blog/where-is-the-diversity-in-applied-behavior-analysis

Conners, B. M. (2020b). Multiculturalism and diversity issues in applied behavior analysis [Unpublished doctoral dissertation]. Seton Hall University.

Covenant 15:16. (2019, June 12). The current status of African Americans within the field of applied behavior analysis. https://www.covenant1516.org/blogs/2019/6/6/the-current$\underline{\text { status-of-african-americans-within-the-field-of-applied-behavior-analysis }}$

C.S. Mott Children's Hospital. (2020). Top health concerns for kids in 2020 during the pandemic. http://mottpoll.org/reports/top-health-concerns-kids-2020-during-pandemic

Data USA. (n.d.). Applied behavior analysis. https://datausa.io/profile/cip/applied-behavior$\underline{\text { analysis }}$ 
BEHAVIOR ANALYSIS AND ANTI-RACISM

Dennison, A., Lund, E. M., Brodhead, M. T., Mejia, L., Armenta, A., \& Leal, J. (2019).

Delivering home-supported applied behavior analysis therapies to culturally and linguistically diverse families. Behavior Analysis in Practice, 12(4), 887-898. https://doi.org/10.1007/s40617-019-00374-1

Disabled World. (2013, September 1). Police violence and people with disabilities. https://www.disabled-world.com/editorials/cops.php

Edbuild. (n.d.). Nonwhite school districts get $\$ 23$ billion less than white districts despite serving the same number of students. https://edbuild.org/content/23-billion\#IL

Equal Justice Initiative. (n.d.). Our work. https://eji.org

Estrada, M., Burnett, M., Campbell, A. G., Campbell, P. B., Denetclaw, W. F., Gutiérrez, C. G., Hurtado, S., John, G. H., Matsui, J., McGee, R., Moses Okpodu, C., Robinson, T. J., Summers, M. F., Werner-Washburne, M., \& Zavala, M. (2016). Improving underrepresented minority student persistence in STEM. CBE-Life Sciences Education, 15(5), 1-10. https://doi.org/10.1187/cbe.16-01-0038

Fisher-Borne, M., Cain, J. M., \& Martin, S. L. (2015). From mastery to accountability: Cultural humility as an alternative to cultural competence. Social Work Education, 34(2), 165181. https://doi.org/10.1080/02615479.2014.977244

Flores, G., Mendoza, F. S., DeBaun, M. R., Fuentes-Afflick, E., Jones, V. F., Mendoze, J. A., Raphael, J. L., \& Wang, C. J. (2019). Keys to academic success for under-represented minority young investigators: Recommendations from the Research in Academic Pediatrics Initiative on Diversity (RAPID) national advisory committee. International Journal for Equity in Health, 18(93), 1-15. https://dpoi.org/10.1186/s12939-019-0995-1 
BEHAVIOR ANALYSIS AND ANTI-RACISM

Fong, E. H., Ficklin, S., \& Lee, H. Y. (2017). Increasing cultural understanding and diversity in applied behavior analysis. Behavior Analysis: Research and Practice, 17(2), 103-113. https://dx.doi.org/10.1037/bar0000076

Glenn, S. S. (2004). Individual behavior, culture, and social change. The Behavior Analyst, 27(2), 133-151. https://doi.org/10.1007/BF03393175

Goode., T. D. (1989). Self-assessment checklist for personnel providing services and supports to children and youth with special health needs and their families. Georgetown University Center for Child and Human Development. https://nccc.georgetown.edu/documents/ ChecklistEIEC.pdf

Gourdine, R. M., \& Algood, C. L. (2014). Autism in the African American population. In V. Patel, V. Preedy, \& C. Martin (Eds.), Comprehensive guide to autism (pp. 2455-2467). Springer. https://doi.org/10.1007/978-1-4614-4788-7 155

Griffin, K. A., \& Muñiz, M. M.(2011). The strategies and struggles of graduate diversity officers in the recruitment of doctoral students of color. Equity \& Excellence in Education, 44(1), 57-76. https://doi.org/10.1080/10665684.2011.540961

Henry, F. (2004, December). Concepts of race and racism and implications for OHRC policy. Ontario Human Rights Commission. http://www.ohrc.on.ca/en/race-policy-dialoguepapers/concepts-race-and-racism-and-implications-ohrc-policy

Hubler, S., \& Bosman, J. (2021, March 11). A crisis that began with an image of police violence keeps providing more. The New York Times. https://www.nytimes.com/2020/06/05/us/police-violence-george-floyd.html

Jackson, G. G. (1976). Is behavior therapy a threat to black clients? Journal of the National Medical Association, 68(5), 362-367. 
BEHAVIOR ANALYSIS AND ANTI-RACISM

Jones, S. H., St. Peter, C. C., \& Ruckle, M. M. (2020). Reporting of demographic variables in the Journal of Applied Behavior Analysis. Journal of Applied Behavior Analysis, 9999, 1-12. https://doi.org/10.1002/jaba.722

Kang, H. K., \& Garran, A. M. (2018). Microaggressions in social work classrooms: Strategies for pedagogical intervention. Journal of Ethnic \& Cultural Diversity in Social Work, 27(1), 4-16. https://doi.org/10.1080/15313204.2017.1413608

Kineavy, F. (2018, October 7). Police violence against people with disabilities continues. Diversity Inc. https://www.diversityinc.com/police-brutality-people-with-disabilities/

Kendi, I. X. (2016). Stamped from the beginning: The definitive history of racist ideas in America. Nation Books.

Kunze, M., Drew, C., Machalicek, W., Safer-Lichtenstein, J., \& Crowe, B. (2019). Language preference of a multilingual individual with disabilities using a speech generating device. Behavior Analysis in Practice, 12(4), 777-781. https://doi.org/10.1007/s40617-019$\underline{00379-W}$

Leland, W., \& Stockwell, A. (2019). A self-assessment tool for cultivating affirming practices with transgender and gender-nonconforming (TGNC) clients, supervisees, students, and colleagues. Behavior Analysis in Practice, 12, 816-825. https://doi.org/10.1007/s40617$\underline{019-00375-0}$

Leonard, J., Horvat, E. M., \& Riley-Tillman, C. T. (2002, April 1-5). Achieving diversity in academia: A dream deferred? [Paper presentation]. Annual Meeting of the American Educational Research Association, New Orleans, LA, United States.

Levy, S. (2019, May 23-27). Social validity in the applied clinical setting: Making change that matters. In S. Levy (Chair), A deeper examination of social validity and its role in 
BEHAVIOR ANALYSIS AND ANTI-RACISM

clinical practice [Symposium]. Association for Behavior Analysis International $45^{\text {th }}$

Annual Convention, Chicago, IL, United States.

Levy, S., Vaidya, J., Dettmering, J., Siebold, A. N., Mittelman, C., \& Garner, J. (2019).

Addressing social and global issues: Viewing the Syrian refugee crisis through a behavior-analytic lens. Behavior and Social Issues, 28, 77-98. https://doi.org/10.1007/ $\underline{\mathrm{s} 42822-019-00018-7}$

Li, A., Wallace, L., Ehrhardt, K. E., \& Poling, A. (2017). Reporting participant characteristics in intervention articles published in five behavior-analytic journals, 2013-2015. Behavior Analysis: Research and Practice, 17(1), 84-91. https://dx.doi.org/10.1037/bar0000071

Li, A., Curiel, H., Pritchard, J., \& Poling, A. (2018). Participation of women in behavior analysis research: Some recent and relevant data. Behavior Analysis in Practice, 11(2), 160-164. https://doi.org/10.1007/s40617-018-0211-6

Liu, J. (2020, June 15). Companies are speaking out against racism, but here's what it really looks like to lead an anti-racist organization. Consumer News and Business Channel. https://www.cnbc.com/2020/06/15/what-it-means-to-be-an-anti-racist-company.html

Lui, P. P., \& Quezada, L. (2019). Microaggressions: What they are, and how they are associated with adjustment outcomes. American Psychological Association. https://www.apa.org /pubs/highlights/spotlight/issue-133

Martin, T. (2018, January 12). Racism 101: Tone policing. Medium. https://medium.com/@tess introvert/racism-101-tone-policing-92481c044b6a

Matsuda, K., Garcia, Y., Catagnus, R., \& Brandt, J. A. (2020). Can behavior analysis help us understand and reduce racism? A review of the current literature. Behavior Analysis in Practice, 13, 336-347. https://doi.org/10.1007/s40617-020-00411-4 
BEHAVIOR ANALYSIS AND ANTI-RACISM

McSweeney, F. K., Donahoe, P., \& Swindell, S. (2000). Women in applied behavior analysis.

The Behavior Analyst, 23(2), 267-277. https://doi.org/10.1007/BF03392015

Meadows, T. (n.d.). A look at diversity \& behavior analysis. I Love ABA! https://www.iloveaba. com/2020/06/a-look-at-diversity-behavior-analysis.html

Mental Health America. (n.d.). Alternatives to calling the police in a crisis.

https://screening.mhanational.org/content/alternatives-calling-police-crisis

Merriam-Webster. (n.d.). Race. In Merriam-Webster.com dictionary. Retrieved July 9, 2020, from https://www.merriam-webster.com/dictionary/race

Metivier, K. (2020, July 2). Envisioning higher education as antiracist. Inside Higher ED. https://www.insidehighered.com/views/2020/07/02/actions-higher-ed-institutions-shouldtake-help-eradicate-racism-opinion

Miller, J., \& Garran, A. M. (2017). Racism in the United states: Implications for the helping professions (2nd ed.). Springer Publishing Company, LLC.

Mittleman, C. (2019, May 23-27). Social validity: What it is and why we need it. In S. Levy (Chair), A deeper examination of social validity and its role in clinical practice [Symposium]. Association for Behavior Analysis International 45 ${ }^{\text {th }}$ Annual Convention, Chicago, IL, United States.

National Association of Social Workers. (2017). Read the code of ethics. https://www.social workers.org/About/Ethics/Code-of-Ethics/Code-of-Ethics-English

National Association of Social Workers. (2020). Where we stand on racism. https://www.social $\underline{\text { workers.org }}$

Oluo, I. (2018). So you want to talk about race. Seal Press. 
BEHAVIOR ANALYSIS AND ANTI-RACISM

Perception Institute. (2014). The science of equality, volume 1: Addressing implicit bias, racial anxiety, and stereotype threat in education and health care. http://perception.org/wpcontent/uploads/2014/11/Science-of-Equality.pdf

Prasad, S. J., Nair, P., Gadhvi, K., Barai, I., Saleem Danish, H., \& Philip, A. B. (2016). Cultural humility: Treatment the patient, not the illness. Medical Education Online, 31(30908), 1 -2 .

Pritchett, M., Ala'i, S., Cruz, A. R., \& Cihon, T. (2020). Social justice is the spirit and aim of an applied science of human behavior: Moving from colonial to participatory research practices. PsyArXiv. https://doi.org/10.31234/osf.io/t87p4

Rosenberg, N. E., \& Schwartz, I. S. (2019). Guidance or compliance: What makes an ethical behavior analyst? Behavior Analysis in Practice, 12, 473-482. https://doi.org/10.1007/ $\underline{\text { s40617018-00287-5 }}$

Sablich, L. (2016, June 6). 7 findings that illustrate racial disparities in education. Brookings. https://www.brookings.edu/blog/brown-center-chalkboard/2016/06/06/7-findings-thatillustrate-racial-disparities-in-education/

Samuel, D. (2020, June 15). Calling the cops on someone with mental illness can go terribly wrong. Here's a better idea. Vox. https://www.vox.com/futureperfect/2019/7/1/ 20677523/mental-health-police-cahoots-oregon-oakland-sweden

Sellers, T. P., Carr, J. E., \& Nosik, M. R. (2020). On the BACB's ethics requirements: A response to Rosenberg and Schwartz (2019). Behavior Analysis in Practice, 13, 714-717. https://doi.org/10.1007/s40617-020-00463-6

Severini, K. E., Ledford, J. R., \& Robertson, R. E. (2018). Systematic review of problem behavior interventions: Outcomes, demographics, settings. Journal of Autism and 
BEHAVIOR ANALYSIS AND ANTI-RACISM

Developmental Disorders, 48(10), 3261-3272. https://doi.org/10.1007/s10803-018-3591$\underline{0}$

Skinner, B. F. (1953). Science and human behavior. Free Press.

Skinner, B. F. (1987). Why we are not acting to save the world. In B. F. Skinner (Ed.), Upon further reflection (pp. 1-14). Prentice Hall.

Sriman, P., \& Klapatch Totsch, J. (2019, May 23-27). Social validity in the applied setting: Where culture and diversity matter. In S. Levy (Chair), A deeper examination of social validity and its role in clinical practice [Symposium]. Association for Behavior Analysis International $45^{\text {th }}$ Annual Convention, Chicago, IL, United States.

Students of Color Caught in the Homework Gap. (n.d.). https://futureready.org/wpcontent/uploads/2020/07/HomeworkGap_FINAL7.20.2020.pdf

Szabo, T. (2020). Equity and diversity in behavior analysis: Lessons from Skinner (1945). Behavior Analysis in Practice, 13, 375-386. https://doi.org/10.1007/s40617-020-00414-1

Tervalon, M., \& Murray-García, J. (1998). Cultural humility versus cultural competence: A critical distinction in defining physician training outcomes in multicultural education. Journal of Health Care for the Poor and Underserved, 9(2), 117-125. https://doi.org/ 10.1353/hpu.2020.0233

Tesfaye, A. (n.d.). BIPOC students and bias in higher education: The necessity of unlearning biases to optimize outcomes for BIPOC students. Payback. https://www.packback.co/stories/bipoc-students-and-bias-in-higher-education-thenecessity-of-unlearning-biases-to-unify-teaching-practices-and-optimize-outcomes-forbipoc-students/ 
BEHAVIOR ANALYSIS AND ANTI-RACISM

U.S. Department of Education. (2016). Advancing diversity and inclusion in higher education. https://www2.ed.gov/rschstat/research/pubs/advancing-diversity-inclusion.pdf

Valentine, P. (2015). Achieving diversity in student enrollment and faculty recruitment. Journal of Best Practices in Health Professions Diversity, 8(2), II-III.

Valenzuela, J., Crosby, L. E., \& Harrison, R. R. (2020). Commentary: Reflections on the COVID-19 pandemic and health disparities in pediatric psychology. Journal of Pediatric Psychology, 45(8), 839-841.

Vandermeer, N. (2020, November 10). OPP find success with mental health response team in eastern Ontario. CTV News. https://ottawa.ctynews.ca/opp-find-success-with-mentalhealth-response-team-in-eastern-ontario-1.5182738

Vela, M. B., Kim, K. E., Tang, H., \& Chin, M. H. (2010). Improving underrepresented minority medical student recruitment with health disparities curriculum. Journal of General Internal Medicine, 25(2), 82-85. https://doi.org/10.1007/s11606-010-1270-8

Vendor Resource Management University. (2016). Diversity and inclusion self-assessment: Office of minority and women inclusion (OMWI) proposed standards summary and selfassessment guide. https://vrmuniversity.com/wpcontent/uploads/2016/11/DISelfAssess mentGuide.pdf

Vincent, J. (2020, June 2). Blackout Tuesday posts are drowning out vital information shared under the BLM hashtag. The Verge. https://www.theverge.com/2020/6/2/21277852/ blackout-tuesday-posts-hiding-information-blm-black-lives-matter-hashtag

Wilhite, C. (2015, November 3). Behavior analysis and the diversity issue. Behavioral Science in the 21st Century. https://bsci21.org/behavior-analysis-and-the-diversity-issue/ 
BEHAVIOR ANALYSIS AND ANTI-RACISM

Williams, D. R. (2018). Stress and the mental health of populations of color: Advancing our understanding of race-related stressors. Journal of Health and Social Behavior, 59(4), 466-485. https://doi.org/10.1177/0022146518814251

Williams, D., \& Sgueglia, K. (2020, November 11). New York City will send mental health teams instead of police to respond to some 911 calls. $C N N$. https://www.cnn.com/2020/ 11/11/us/new-york-city-mental-health-911-trnd/index.html

Worland, J. (2020, June 11). America's long overdue awakening to systemic racism. Time. https://time.com/5851855/systemic-racism-america/

Wright, P. I. (2019). Cultural humility in the practice of applied behavior analysis. Behavior Analysis in Practice, 12, 805-809. https://doi.org/10.1007/s40617-019-00343-8 


\section{Appendix A}

Vignette - African American Vernacular English (AAVE)

You work as an RBT with an African American child who has specific communication goals (i.e., expressive skills). During one of your sessions, the child says " $\mathrm{X}$," which is outside the current criteria for correct responding as outlined/suggested by based on a program developed from the ABLLS-R. Having worked with this family for a while, you identified/noticed that this response is consistent with what you have heard other family members say. How would you proceed?

- Incorrect RBT's response: Correct their response to make sure it matches criteria and consult with your supervisor.

- Correct proactive RBT's response: Consistent with my training in cultural humility, I spoke with the family to outline and identify common communicative phrases that would likely be reinforced in their natural environment before targeting communication skills with this client. Given that the response emitted by the client was identified as a correct response, social praise is delivered.

- Correct reactive RBT's response: Given my training in cultural humility, I understand that this child is actually bilingual and is speaking AAVE. As a result, this is the language that is likely to be reinforced in their natural environment and the RBT records the client's response as correct.

- Points for potential discussion for instructors and students

- Cultural humility

- AAVE and bilingualism

0 How to adapt common assessments (e.g., ABLLS-R) when working with diverse populations

- Social validity 
BEHAVIOR ANALYSIS AND ANTI-RACISM

\section{Appendix B}

\section{Useful Resources}

- Covenant 15:16 (2019) - https://www.covenant1516.org/blogs/2019/6/6/the-current$\underline{\text { status-of-african-americans-within-the-field-of-applied-behavior-analysis }}$

- Black Applied Behavior Analysts, Inc. (n.d.) - https://www.babainfo.org/

- $\quad$ Equal Justice Initiative (n.d.) - https://eji.org

- Kendi (2016) - https://www.ibramxkendi.com/antiracism-center-2

- Cochran (2018) - https://scholarlykitchen.sspnet.org/2018/06/22/problem-diversityinclusion-equity/

- Goode (1989) - https://nccc.georgetown.edu/documents/ChecklistEIEC.pdf

- Vendor Resource Management University (2016) - https://vrmuniversity.com/wpcontent/uploads/2016/11/DISelfAssessmentGuide.pdf

- Beautiful Humans Change (2020) - https://www.beautifulhumanschange.com/resources 